\title{
LA PRODUCCIÓN QUESERA EN EL NOROESTE DE CHIHUAHUA: EL QUESO TRADICIONAL MENONITA
}

ÓSCAR ARTURO SÁNCHEZ CARLOS

ELIZABETH BAUTISTA FLORES

\section{RESUMEN}

1 1 queso menonita de Chihuahua es un producto típico local. Con el tiempo, se ha convertido en un producto donde se fincan un origen, una cultura y un alimento agroalimentario, que expresa su calidad y arraigo con base en la geografía y las prácticas culturales. De ahí que sea necesario hacer una reconstrucción de la historia para subrayar la importancia de los queseros, conocer la distribución industrial, identificar el perfil del consumidor, así como la incorporación de este alimento en la dieta diaria de los chihuahuenses. Este texto incluye como ejemplo el caso de la región noroeste de Chihuahua.

Palabras clave: agroindustria quesera, noroeste de Chihuahua, queso tradicional, menonitas.

\section{INTRODUCCIÓN}

La producción industrial del queso ha evolucionado de manera tal que se combinan variables como la tradición con la tecnología; el interés por conservar la identidad alimentaria con las nuevas exigencias del mercado y la cultura conservadora con las nuevas 
tendencias de los consumidores, todo ello dentro de un escenario de globalización.

En Chihuahua se encuentran asentadas diferentes colonias de menonitas, que son un grupo étnico-religioso, derivado del anabaptismo europeo del siglo XVI. A México llegaron en 1922 y desde entonces han tenido un desarrollo económico, principalmente con base en actividades agroindustriales, donde destaca el queso, el cual es conocido como menonita.

El queso menonita se produce en varias colonias del estado de Chihuahua, siendo los de mayor penetración en el mercado los elaborados en las industrias asentadas en el municipio de Cuauhtémoc, donde se ha pasado de la tradición a la industrialización. En el noroeste de Chihuahua, la producción es de menor escala y las queserías son pequeñas, pues ninguna alcanza los veinte empleados y es común que carezcan de cadenas de producción y distribución para el queso. Estas se ubican en municipios como Janos, Ascensión y Nuevo Casas Grandes.

Los objetivos de este texto son reconstruir la o las historias que se tienen sobre el origen del queso menonita en Chihuahua y, con ello, sentar las bases para el análisis para comprender la subjetividad alimentaria que existe entre los consumidores de queso menonita, tanto de las localidades cercanas a la región de Casas Grandes como de Ciudad Juárez. Para ello, se identifica el perfil del consumidor del queso menonita y, por último, conocer los parámetros de arraigo que existen entre los consumidores, así como la apuesta comercial que miembros de las colonias menonitas están implementando para generar dinámicas de desarrollo en espacios rurales de manera interna.

Por lo anterior, se formulan las siguientes interrogantes: ¿cómo el queso menonita puede contribuir a la construcción de subjetividades en Chihuahua?, ¿cuáles son las características agroindustriales que se muestran en el noroeste de Chihuahua, a partir de la producción del queso menonita?, ¿cuál es el nivel de consumo del queso menonita en la región? y ¿cuáles son las oportunidades de venta en el mercado local para el queso menonita en el estado de Chihuahua? 
Por lo tanto, el documento se encuentra integrado por cuatro partes. La primera explica la importancia del queso y la relación con el territorio, para luego exponer las narrativas que se han recuperado para comprender el origen del queso menonita en Chihuahua, pues pocas son las referencias que se tienen y los relatos difieren; así se comprenderán las formas organizacionales de las colonias y su adopción en la gastronomía chihuahuense. A lo largo de ese apartado, cabe mencionar que es importante mostrar un poco de la discusión que existe para hacer una distinción entre lo que es el queso menonita y el queso chihuahua, pues aunque son parecidos cada uno presenta características particulares.

La segunda parte explica cómo se han organizado las queserías en el noroeste del estado, cuáles son las colonias donde se ubican, así como los destinos de venta del producto. En esta parte se analizará la cadena de producción, donde quedan evidenciadas la falta de estrategias de negocios, pues aunque se tiene un buen producto y existe una alta demanda en el mercado regional, se carece de directrices empresariales.

La tercera parte exhibe los resultados que se obtuvieron de la utilización de, al menos, tres instrumentos cuali-cuantitativos diferentes, los cuales se aplicaron entre abril de 2016 y febrero de 2017, tanto para conocer la importancia del queso menonita en el mercado regional como para evidenciar algunas subjetividades que los consumidores han construido a partir de este alimento, que es prácticamente imprescindible en la dieta diaria de los chihuahuenses en el noroeste. Por último, se añaden las conclusiones a las que se llegaron.

\section{EL QUESO MENONITA: TERRITORIO, CULTURA Y GASTRONOMÍA}

De acuerdo con la literatura científica, los productos agroalimentarios típicos representan la peculiaridad de un territorio, de sus recursos, de sus conocimientos, de sus relaciones sociales, de su historia y de su cultura (Belletti, Brunori, Marescotti, Pacciani, \& Rossi, 2006). Es decir, son la expresión de un sistema agroali- 
mentario "localizado" que se ha desarrollado sobre la base del uso de los recursos específicos locales (Velarde, 2012).

En ese sentido, el producto típico se diferencia de otros de calidad específica (productos orgánicos, por ejemplo), porque el o los productos típicos son el resultado de la interacción entre diversos productores locales que pertenecen y forman parte de una comunidad, además de que incorporan una serie de saberes construidos a lo largo del tiempo, los cuales son parte de una colectividad territorializada (Benavente, 2008). Estos factores lo convierten en un patrimonio de la comunidad local, que es la única que puede legítimamente apropiarse de los beneficios económicos, sociales y culturales que implica el proceso mismo de valorización como producto con identidad territorial (Belletti, 2003).

De acuerdo con el Programa de Desarrollo de la Agroindustria Rural de América Latina y el Caribe (Prodar) con sede en el Instituto Interamericano de Cooperación para la Agricultura (IICA) - Perú, citado por Ranaboldo et al. (2010), se mencionan las características de la agroindustria en América Latina, entre las cuales se destacan las siguientes:

Aspectos tecnológicos: se trata de una transformación heredada o autoaprendida; tecnologías tradicionales, heredadas, con baja productividad; manejo deficiente del producto y un alto esfuerzo físico.

Características operacionales: la mayoría de las empresas son individuales o familiares y normalmente trabajan con procesos simples; en este tipo de organizaciones, la actividad es complementaria al ingreso de la familia.

La producción de queso, de acuerdo con lo que indican Villegas y Cervantes (2011), va más allá de la cuajada de leche, pues en el mejor de los casos se refiere a un "bien cultural alimentario", el cual da orden y sentido a toda una red o cadena alimentaria local, que por supuesto tiene resonancia en los niveles económicos y sociales. Por lo tanto, es necesario considerar que cuando se habla de quesos genuinos artesanales, se pueden considerar como elementos esenciales aquellos recursos propios del territorio, como son:

- Un saber hacer tradicional, patrimonializado en el sentido de que se transmite dentro de una comunidad "localizada". 
Este saber hacer puede generar propiedades organolépticas específicas.

- Una reputación vinculada a una región reconocida como productora de un queso particular con determinada calidad (sensorial y/o identitaria), la cual la torna intransferible al saber hacer, territorializándolo.

- Una calidad de leche y de microflora láctica vinculada a los recursos naturales y con un saber hacer ganadero. En conjunto con esas prácticas, se origina una serie de otros bienes que tienen características patrimoniales: paisajes, cultura, organización social, etcétera.

- Una cultura de producción y uso del queso.

Saberes relacionales que permiten el buen funcionamiento de la cadena productiva (Villegas, \& Cervantes, 2011, p. 150).

En México existen muchos casos donde la producción de queso no es la actividad principal del ingreso familiar, pero sí representa arraigo y fortalecimiento de la tradición. Por lo tanto, la elaboración de quesos, puede afirmarse que posee un profundo arraigo territorial y reconocimiento de los consumidores, ya que la sociedad registra el patrimonio cultural e histórico de los productores y se reconoce implícitamente la transmisión de los saberes de su elaboración de generación en generación. Además, los consumidores del queso recogen la esencia de los productos en las regiones de origen en donde está plasmado el sabor que lo hace único, aspecto que lo presenta atractivo al consumidor, quien entiende que detrás del producto se encuentran los valores de los productores.

Este también puede ser el caso de lo que sucede en las colonias menonitas, ya que una gran cantidad de colonos tiene ganado lechero y el lácteo es canalizado hacia las fábricas de queso, las cuales están constituidas como cooperativas familiares o sociedades de producción rural. De ahí la importancia de analizar las aportaciones de la producción láctea en una región como la de Casas Grandes, que para fines de este texto comprende los 
municipios de Ascensión, Buenaventura, Janos y Nuevo Casas Grandes.

\subsection{Origen del queso menonita: las historias}

El 1 de marzo de 1922 salió de Manitoba, Canadá, el primero de los seis trenes que contrataron los menonitas, con un costo de treinta mil dólares cada uno, para transportarlos hasta México. Su destino fue San Antonio de los Arenales. El día 8 del mismo mes arribaron 9263 personas (Llera, \& Bautista, 2013). Cada familia traía, además de sus pertenencias personales, su menaje de casa, carros de transporte, caballos de tiro, vacas lecheras, pollos, gansos, cerdos, implementos agrícolas, semillas para siembra, maderas y materiales para la construcción de sus casas, así como la cantidad de quince millones de pesos. Se organizaron en dos colonias: Manitoba, conformada por campos numerados del 1 al 42, y Swift Current, conformada por campos numerados del 101 al 117. Las tierras fueron adquiridas legalmente y distribuidas con alrededor de 40 acres por familia.

Sin embargo, a pesar de casi un siglo de realizarse los primeros asentamientos menonitas, aún se desconoce con precisión el origen de este alimento lácteo. Hasta el momento de estas investigaciones, se han logrado recuperar, al menos, tres historias diferentes que tratan de explicar el origen del queso menonita. Estas son producto de narraciones que se tienen dentro de las propias colonias y no se han documentado propiamente.

Una primera historia refiere que la actividad de transformación láctea, es prácticamente inherente a los menonitas, pues su origen se remonta a la antigua Frisia, hoy Holanda, pues desde sus primeros años se asentaron en grandes planicies y praderas, donde la producción de leche era uno de sus principales ingresos y de ahí su experiencia en materia de transformación en productos como mantequilla, queso, crema, por mencionar algunos.

Por lo tanto, el trabajo con ganado lechero es común entre la colonia menonita, por lo que la producción de queso no les es un 
tema ajeno. Sin embargo, debieron enfrentar los desplazamientos constantes de sus colonias, debido a las persecuciones y defensa de su fe. De ahí que algunos entrevistados indiquen que, al poco tiempo de llegar a tierras mexicanas, comenzaron con la producción de este alimento, primero como autoconsumo y luego en la presentación de ruedas de 3 a 5 kilos; posteriormente fabricaron el producto en forma de barras, lo cual tuvo una gran aceptación en el mercado mexicano.

Una segunda historia se remonta a la producción de queso y a las técnicas rústicas de su elaboración. De acuerdo con una entrevista con Abraham Peters, quien también es conocido como cronista de las colonias menonitas en Cuauhtémoc, indicó que el primer queso que se produjo fue en el campo 6A de la colonia Manitoba, ubicada en ciudad Cuauhtémoc, en la casa de Jacobo Wibe.

De acuerdo con este relato, se menciona a don Luis Lara Leos, quien fue un comerciante que viajaba por varias ciudades de la entidad, como Nuevo Casas Grandes, Cuauhtémoc y Ciudad Juárez, para vender dulces y chocolates en la región menonita. Debido a las constantes visitas a Nuevo Casas Grandes, don Luis tenía una muy buena relación con la comunidad mormona asentada en la colonia Dublán ${ }^{1}$ en esa región. Al llegar a la región menonita, le llamó la atención la producción de queso con una técnica rudimentaria, pero de muy buena calidad.

A él se le atribuye la idea de elaborar un queso con una técnica y calidad que pudiera ser aceptado por toda la comunidad chihuahuense, y con ello ser comercializado a mayor escala. Para tal efecto, pidió ayuda a un amigo de origen mormón, apellidado Cole, quien también vivía en la colonia Dublán. Luego de un reco-

1 La colonia Dublán es una de tres colonias donde se establecieron mormones a fines del siglo XIX. Esta, junto con la colonia Juárez, se mantienen como puntos de interés para los turistas, debido al diseño de sus calles y fina arquitectura. La colonia Porfirio Díaz desapareció sin que haya indicios de su ubicación. Todas ellas se instalaron en el noroeste de Chihuahua como una forma de estimular la agricultura intensiva, debido a los, entonces, novedosos sistemas de riego. El nombre lo adoptan en honor a Manuel Dublán (1830-1891), ministro de Hacienda durante el gobierno de Porfirio Díaz (1880 a 1910). Actualmente existe como zona residencial y es parte de la ciudad de Nuevo Casas Grandes, Chihuahua. 
rrido por la zona menonita y encontrando una buena disposición de los mismos, acordaron instalar una fábrica de queso elaborado con una técnica mixta, compuesta por la holandesa de los menonitas y la estadounidense de los mormones. De esta manera, en 1936, a decir de Abraham Peters, se creó lo que ahora conocemos como queso menonita.

Este intercambio de conocimientos entre mormones y menonitas, podría explicar que en la década de 1940 se instalara en ciudad Cuauhtémoc una tienda que vendía quesos y llevaba el nombre de "Quesería Dublán", misma que, al paso de los años, tuvo problemas económicos, por lo que en la década de 1950 debió cerrar sus operaciones empresariales.

Sin embargo, en esa misma década, cuando ya era conocida y perfeccionada la fabricación de quesos, surgió otra alternativa de negocios: "Quesos Las Pampas", que inició sus operaciones a principios de 1950 como una cooperativa con el liderazgo de Enrique Wall. Esta empresa hasta ahora existe y ha estandarizado todos sus procesos de producción con base en la normatividad sanitaria actual.

La tercera historia señala que un empleado de origen menonita de Chihuahua aprendió la técnica de un farmacéutico alemán, lo cual hizo que pronto se comenzara a considerar como una oportunidad para producir estos quesos (López, \& Vargas, 2011). En lo particular, se considera que esto es poco creíble, pues los menonitas tienen un sentido de trabajo dentro de la comunidad. Ofertar su fuerza de trabajo fuera de la comunidad o para terceros externos a la comunidad, es poco frecuente. Además de que el trabajo individual tampoco es bien aceptado. Dentro de las colonias menonitas existe un sentido comunitario sólido. Por lo tanto, es frecuente que en forma colectiva compartan las ideas, tomen decisiones y cada vez más colonos sumen sus esfuerzos para alcanzar objetivos comunes.

En cualquiera de las tres versiones debe mencionarse que las empresas fueron fincadas en cimientos colectivos o de cooperativas, ya que ningún menonita tenía los recursos suficientes para emprender de manera individual una empresa quesera. 
Lo importante es que los miembros de las colonias menonitas consideran que el queso que producen es similar al queso cheddar, que es de origen europeo; sin embargo, su proceso de elaboración es diferente, pues no alcanza el color amarillo intenso ni la porosidad, debido a los días de maduración de aquel, y tampoco se incluyen colorantes naturales como los pimientos.

Otra acción que ayudó a consolidar la industria quesera menonita en Cuauhtémoc, fue que periódicamente se reunían los administradores de las cooperativas queseras y compartían sus conocimientos, sobre todo aquellos que resultaban en beneficio del negocio, y de esa manera había un crecimiento relativamente similar entre sí, además de que se mantuvo casi la misma técnica de elaboración.

Con el entonces gobernador del estado de Chihuahua, Patricio Martínez García (1998-2004), se realizaron acciones para mejorar las condiciones y tecnología de elaboración de los quesos menonitas; sin embargo, no todas las queserías adoptaron las nuevas disposiciones. Con el apoyo del gobierno estatal, la quesería del campo 70 recibió un gran impulso, siendo asesorada por técnicos europeos para hacer una auténtica reingeniería y capitalizando todos los apoyos gubernamentales para consolidarse como una de las mejores queserías de la región menonita en Cuauhtémoc.

De acuerdo con Abraham Peters (historiador, agricultor, lechero y cooperativista de la Sociedad Cooperativa Bajío de Abajo Queso Pampas), el queso menonita no sólo es el producto representativo de los menonitas, sino que también se constituyó en otro pilar de su economía, ya que en épocas difíciles de sequía, donde el ganado de carne tiende a morir o los cultivos no alcanzan los rendimientos esperados, el queso es aquel que "los saca adelante”, pues por varios años se apoyaron en este producto para disponer de recursos económicos.

\subsection{Producción láctea}

La producción lechera en México tiene un incremento modesto respecto a la demanda nacional por el lácteo, ya que la 
producción de 2011 fue de 10000724000 de litros, mientras que en el año 2016 alcanzó los 11000747000 de litros, de acuerdo con datos oficiales de la Secretaría de Agricultura, Ganadería, Desarrollo Rural, Pesca y Alimentación, así como el Servicio de Información Agroalimentaria y Pesquera (Sagarpa-SIAP, 2017). Esa misma fuente indica que las importaciones de leche ascendieron a 40000 toneladas en 2015, mientras que las importaciones de leche en polvo representaron $53.4 \%$ respecto a la producción nacional.

La Tasa de Crecimiento Media Anual (TCMA) de producción de leche fluida en México, de 2011 a 2015, fue de $1.22 \%$, mientras que la Tcma de leche en el estado de Chihuahua durante el mismo periodo fue de $2.14 \%$, lo cual demuestra un mayor dinamismo en el estado con respecto al crecimiento nacional. Para el año 2016, la industria de los quesos produjo 332000251 toneladas con un valor en el mercado de \$15000 351000 (Sagarpa-SIAP, 2016). De acuerdo con la misma fuente, las principales variedades producidas fueron: fresco, $17 \%$; panela, $15 \%$; doble crema, $13 \%$; Chihuahua, $11 \%$; amarillo, $11 \%$. A nivel estatal, Chihuahua ocupa la cuarta posición en la producción de leche con 1034000227 000 litros, posicionándose por debajo de los estados de Jalisco, Coahuila y Durango (SIAP, 2016).

Por su parte, los datos generados por el Instituto Nacional de Estadística y Geografía (Inegi), a través de la Encuesta Mensual de la Industria Manufacturera (EMIM), tienen registrado una producción de 3307 toneladas de queso chihuahua para febrero de 2015, que probablemente corresponde a la producción de empresas que están en el padrón de la Cámara Nacional de la Industria de Transformación (Canacintra). Sin embargo, no se tiene información del origen de la leche y aspectos relacionados con la oferta y el comportamiento del consumidor de este producto.

Cabe mencionar que para las autoridades sanitarias y de gobierno no está clara la diferencia entre el queso chihuahua y el queso menonita. Según la Procuraduría Federal del Consumidor (Profeco), hay más de doce marcas comerciales diferentes que producen queso chihuahua, aunque solo contabiliza las grandes empresas nacionales y no considera los quesos elaborados por 
queserías locales menonitas y no menonitas, incluidos los producidos en otras entidades del país.

La confusión entre ambos quesos es común. Esta fue acentuada por la autoridad, ya que el 24 de junio de 2011 se publicó la Norma Oficial Mexicana NMX-F-738-COFOCALEC-2011, con el objetivo de establecer reglas claras para la elaboración del queso chihuahua en el país. El Gobierno del Estado, productores, cámaras empresariales, así como instituciones educativas y de investigación, se incorporaron al Subcomité Técnico de Normalización del Consejo para el Fomento de la Calidad de la Leche y sus Derivados, A. C. (Cofocalec), para revisar los procedimientos y estableciendo una norma con especificaciones de control para la fabricación del producto. De esa forma, prácticamente se homologó al queso menonita con el queso chihuahua y, con ello, se dejó de lado todo el conocimiento e historia de cada uno de los productos.

A modo de ejemplo, en tres municipios del noroeste de Chihuahua hay más de once queserías menonitas (Sánchez, Bautista, \& Cervantes, 2016) que no están consideradas en esa estadística del gobierno federal, pero este punto se abordará con mayor detalle en el siguiente apartado.

\subsection{Menonita o chihuahua: el queso del norte}

Como se ha mostrado en el apartado anterior, hablar de menonitas es hablar de quesos. En todo el estado de Chihuahua, ha sido apreciado este producto, ya que los chihuahuenses lo relacionan con los valores de esta comunidad, tales como el trabajo, la sencillez del estilo de vida y, en general, su cultura e historia. Aunque poco se sabe de las diferencias entre el queso menonita y el queso chihuahua, es preciso afirmar que sí existen distinciones.

De tal forma, puede decirse que el queso menonita es poco más ácido al degustarlo, además de ser firme al cortar; con una corteza gruesa de tono amarillento y presenta algunas porosidades en su interior, debido al proceso de maduración. Cabe mencionar que para producir un kilo de queso en promedio, se requieren diez litros de leche. Algunos productores tienen la idea de que al usar 
una mayor cantidad de litros (doce litros de leche), la consistencia del queso será más compacta (menos porosa); sin embargo, la densidad del queso está en función del cultivo enzimático utilizado para el cuajado, así como la presión a la que es sometido en el proceso de prensado, donde se elimina el suero.

Estas variables determinan, en buena medida, el sabor y la consistencia. Mientras que la inocuidad debe ser cuidada desde la sanidad de la vaca, ordeña, manipulación de la leche y todo el proceso de elaboración del queso. Cabe señalar que la raza del ganado bovino es Holstein, debido a que se adapta a las condiciones climáticas de la región, mientras que el sistema de producción es estabulado; en tanto se alimentan de alfalfa, silo forrajero, avena y frecuentemente se utiliza alimento balanceado, que es proporcionado durante la ordeña.

El queso también se llama queso menonita y/o campresino menonita, a propósito de las comunidades menonitas del norte de México. Cabe destacar que campresino, se refiere al procedimiento de compresión utilizado para producir este tipo de queso, similar al queso cheddar (Drake, 2004), y que puede ser rectangular o redondo.

En términos generales, el queso menonita es de consistencia semidura y en su mayoría es producido a partir de leche pasteurizada. Pocas son las familias que, a pesar de las restricciones que impuso la Comisión Federal para la Protección contra Riesgos Sanitarios (Cofepris), en 2012, para estandarizar la producción de queso desde las normas sanitarias, tratan de mantener la receta original con base en el uso de leche cruda de vaca.

Los atributos sensoriales (sabor y textura) son críticos para la identificación del queso y la aceptación del consumidor. A pesar de que muchos enfoques han sido desarrollados, la evaluación sensorial del queso usando el análisis descriptivo como herramienta de investigación, ha florecido en la última década (Drake, 2004).

De acuerdo con la literatura especializada, el establecimiento de los perfiles de sabor y textura para el tradicional queso menonita elaborado en Chihuahua, México, representa un enlace crítico de caracterización en los rasgos de calidad del producto. El queso 
menonita es semiduro y tiene un sabor ligero, a diferencia de los elaborados con leche cruda, donde se percibe un sabor más fuerte que en los elaborados con leche pasteurizada (Van Hekken, Drake, Molina Corral, Guerrero Prieto, \& Gardea, 2006). En ese sentido, podría considerarse más cercano el queso menonita al queso chéster, ya que es más pálido que el cheddar y más suave en el aroma. Al menos en el noroeste de Chihuahua en algunas etiquetas de los quesos sí se indica como tipo chéster.

Por su parte, las características del queso chihuahua son: textura semifirme y color amarillo dorado, a diferencia de muchos otros quesos mexicanos que son blanquizcos. Además, el queso chihuahua muestra un sabor suave y mantecoso, y no tiende a la porosidad del queso menonita, además de que suele ser elástico.

Por sus características, tanto físicas como sensoriales, se ha incrementado su demanda a nivel nacional. Esto ha originado un cambio de producción a la industrial, lo cual implica no sólo conocer las características físicas, sino el comportamiento del consumidor, sobre todo en las poblaciones cercanas a los centros de producción, ya que es un producto que los consumidores perciben que lleva la cultura y los valores que los identifican.

El historiador Jesús Vargas Valdéz, quien es una autoridad en esa disciplina en el estado de Chihuahua, afirma que el queso menonita es el que se fabrica con un promedio de diez litros de leche por kilo de queso, debido al uso de procesos tradicionales que los menonitas han aprendido de generación en generación; incluso, algunos de ellos emplean procesos industriales tecnificados, mientras que el queso chihuahua es el tipo ranchero que requiere quince o dieciséis litros de leche para producir un kilo de queso, y que tiene su origen en el saber hacer, el cual está relacionado con la producción de chihuahuenses mestizos.

\section{QUESERÍAS MENONITAS DEL NOROESTE DE CHIHUAHUA}

El crecimiento poblacional de los asentamientos de las colonias menonitas en la ciudad de Cuauhtémoc, así como el espíritu migrante llevaron a ciudadanos menonitas a asentarse en el noroeste del estado de Chihuahua. 
Así, la primera colonia que se estableció en la región noroeste de Chihuahua fue Buenos Aires, en el municipio de Janos en 1959. El municipio de Janos colinda con el estado de Sonora y con Nuevo México, Estados Unidos. Posteriormente, se instalaron colonias menonitas en otros municipios que comprenden esta región, tales como Ascensión, Buenaventura y Nuevo Casas Grandes.

Actualmente, de las dieciséis colonias que se tienen registradas, la mayoría guardan cierta distancia de los asentamientos urbanos, en buena medida debido a que los colonos profesan un ala de la región menonita un poco más conservadora que las de sus compañeros en Cuauhtémoc.

Así puede comprenderse que la mayoría de los caminos son aún de terracería y que en pocas ocasiones tienen servicio de telefonía. La colonia de mayor desarrollo económico es El Valle, la cual muestra un desarrollo similar al corredor de Manitoba, Cuauhtémoc. Ello, a pesar de haber sido uno de los últimos asentamientos en la región. La colonia El Valle se ubica a la orilla de la carretera federal Chihuahua-Nuevo Casas Grandes y ahora cuenta con calles pavimentadas, alumbrado público, pista para aeronaves, hoteles, restaurantes, tiendas departamentales, escuelas y, por supuesto, iglesias.

El mayor número de queserías se encuentran en la colonia Las Virginias, en el municipio de Janos. En ella, se tiene contabilizada una producción láctea de 25500 litros de leche y cinco queserías en sus nueve campos, al momento de escribir este texto. La colonia El Capulín tiene una producción láctea de 33500 litros por día, además de tres queserías registradas.

Debe señalarse que todos los campos de esta colonia tienen producción lechera, la cual se canaliza a las plantas queseras, donde algunas de ellas presentan un alto grado de desarrollo tecnológico (industria láctea); tan es así, que varias de las queserías tienen equipos con tecnología de punta, el resto utilizan procesos semitecnificados y las menos emplean técnicas artesanales.

Por su parte, la colonia El Sabinal tiene tres queserías, donde una de ellas, la del campo 1, procesa un promedio de 10000 litros de leche diarios utilizando sistemas tecnificados, mientras que las 
otras dos utilizan sistemas semitecnificados. Cabe hacer mención que en esta colonia, las queserías disponen de generadores de electricidad de combustión interna para sus procesos de agitación y enfriamiento, debido a que tienen tradiciones conservadoras y no usan energía eléctrica proporcionada por la Comisión Federal de Electricidad (CFE). El resto de las colonias sí utilizan la energía eléctrica, tanto para sus actividades laborales como para sus hogares, además de que emplean procesos artesanales, semitecnificados y tecnificados.

\subsection{Metodología aplicada}

Esta investigación se desarrolló de abril de 2016 a febrero de 2017 y, para ello, se aplicaron los siguientes instrumentos cualicuantitativos. Para una parte cualitativa, se utilizaron técnicas de entrevistas abiertas y semiestructuradas con personas dedicadas a la producción y comercialización de queso.

En virtud del desconocimiento del número y ubicación de las queserías en la zona de estudio, se visitó cada una de las dieciséis colonias menonitas que se encuentran en los cuatro municipios del noroeste de Chihuahua para comprobar su existencia, así como algunas de sus características. En ese momento, se buscó a los propietarios y/o responsables de cada quesería, para solicitar su apoyo para responder algunas preguntas del cuestionario preparado para ello. Se realizaron once entrevistas a propietarios y se sistematizó la información, lo que permitió diseñar un cuadro de identificación de cada quesería en la región (véase cuadro 1).

Mientras que los datos cuantitativos, se obtuvieron por medio de dos cuestionarios, los cuales se aplicaron principalmente a consumidores. Para ello, se hizo un levantamiento de 256 encuestas a consumidores de quesos, por lo cual se ubicaron ocho puntos de venta en ciudades de Nuevo Casas Grandes, que comprendieron tiendas especializadas en lácteos y algunas de venta directa, así como tiendas departamentales; otro punto se ubicó en la Central de Abasto en Ciudad Juárez, pues la producción de quesos en la región noroeste es poco común que se venda en tiendas especia- 
lizadas o departamentales de aquella ciudad, debido a que en su mayoría carece de etiquetado, marca y otras características que requieren las tiendas de autoservicio.

Cuadro 1. Producción láctea en colonias menonitas del noroeste de Chihuahua.

\begin{tabular}{|c|c|c|c|c|c|c|}
\hline Colonia & \multicolumn{5}{|c|}{ Las Virginias, Municipio de Janos } & \multirow{2}{*}{$\begin{array}{c}\text { Total de } \\
\text { leche (Lts) }\end{array}$} \\
\hline Campo & Campo 1 & Campo 3 & Campo 6 & Campo 7 & Campo 9 & \\
\hline $\begin{array}{c}\text { Producción } \\
\text { (Lts leche/día) }\end{array}$ & 2000 & 5000 & 11000 & 4500 & 3000 & 25500 \\
\hline Colonia & \multicolumn{5}{|c|}{ El Cuervo, Municipio de Janos } & \multirow{2}{*}{$\begin{array}{c}\text { Total de } \\
\text { leche (Lts) }\end{array}$} \\
\hline Campo & Campo 26 & & & & & \\
\hline $\begin{array}{c}\text { Producción } \\
\text { (Lts leche/día) }\end{array}$ & 3000 & & & & & 3000 \\
\hline Colonia & \multicolumn{5}{|c|}{ Buenos Aires, Municipio de Janos } & \multirow{2}{*}{$\begin{array}{c}\text { Total de } \\
\text { leche (Lts) }\end{array}$} \\
\hline Campo & Campo 1 & & & & & \\
\hline $\begin{array}{c}\text { Producción } \\
\text { (Lts leche/día) }\end{array}$ & 4000 & & & & & 4000 \\
\hline Colonia & \multicolumn{5}{|c|}{ El Capulín, Municipio de Casas Grandes } & \multirow{2}{*}{$\begin{array}{c}\text { Total de } \\
\text { leche (Lts) }\end{array}$} \\
\hline Campo & Campo 2 & Campo 7 & Campo 9 & & & \\
\hline $\begin{array}{c}\text { Producción } \\
\text { (Lts leche/día) }\end{array}$ & 8000 & 2500 & 500 & & & 25500 \\
\hline Colonia & \multicolumn{5}{|c|}{ El Sabinal, Municipio de Ascensión } & \multirow{2}{*}{$\begin{array}{c}\text { Total de } \\
\text { leche (Lts) }\end{array}$} \\
\hline Campo & Campo 3 & Campo 4 & Campo 7 & & & \\
\hline $\begin{array}{c}\text { Producción } \\
\text { (Lts leche/día) }\end{array}$ & 2000 & 5000 & 11000 & & & 16000 \\
\hline & & & & Total & lect & 82000 \\
\hline
\end{tabular}

Fuente: elaboración propia con datos obtenidos en trabajo de campo (2017).

El primer instrumento para los consumidores fue para identificar su perfil, así como las tendencias del consumo y la identificación del producto. Para ello, se diseñó un instrumento cualicuantitativo con un total de treinta preguntas. De ellas, veinticinco fueron de opción múltiple y tres, se incluyeron en una matriz de dos entradas, para conocer el nivel de compra, degustación y preferencia de marca. Solo dos preguntas fueron abiertas, para que se recopilaran datos de forma libre. 
El segundo cuestionario cuali-cuantitativo fue en relación con elementos sensoriales (degustación y subjetividad). Este incluyó quince preguntas; ocho fueron cerradas y siete, abiertas, a fin de recopilar la respuesta inmediata del consumidor. Se incluyó una matriz de dos entradas, para hacer los comparativos entre dos tipos de quesos: queso 1 (chihuahua) y queso 2 (menonita), donde se solicitó a las personas que señalaran las características en cuanto a color, olor, textura y sabor, así como comprender los elementos subjetivos en relación con el producto de origen lácteo. Con base en la escala de Likert, que incluye parámetros de 0 a 10, se solicitó calificar a cada queso en cuanto a mascabilidad, suavidad y preferencia.

En ella, se buscó conocer los niveles sensoriales para diferenciar el queso menonita del queso chihuahua, pues en el primer instrumento se afirmó que más de la mitad de la población encuestada, en ese momento, conocía la diferencia entre uno y otro.

Posteriormente, se sistematizaron los datos obtenidos a partir de una base de datos generada en el software SPSS, el cual ayudó a la visualización de la información donde se muestran las tendencias de consumo y oportunidades de negocio para los productores.

\section{RESULTADOS}

De acuerdo con las entrevistas realizadas a algunos productores de queso, se indicó que la producción regional de queso menonita se canaliza principalmente a Ciudad Juárez, mientras que $10 \%$ de esa producción, se destina al mercado local de las cabeceras municipales de Nuevo Casas Grandes, Ascensión, Janos y Buenaventura.

Cabe mencionar que de las empresas que existen, ninguna tiene más de quince empleados, por lo que se consideran microempresas o empresas familiares, pues en la mayoría de los casos es la propia familia (padres e hijos) quien se encarga de todo el proceso de elaboración, comercialización y venta del producto. Además, algunas de ellas no tienen un registro de marca y, por lo tanto, carecen de etiquetado. 
El $70 \%$ de los propietarios, y que, además, son los administradores y laboran en las queserías, tienen entre treinta y cincuenta años de edad, lo que indica que, en general, es gente joven, por lo que es más fácil explicar el dinamismo de la actividad quesera en los diferentes asentamientos.

Un aspecto importante fue conocer cuáles son los motivos para desarrollar la actividad de producir queso, encontrando que $73 \%$ lo hacen porque así les enseñaron sus padres, es decir, es una herencia, y también por gusto, hecho que consolida mantener el saber hacer entre la comunidad menonita.

En cuanto al parentesco de los trabajadores con los propietarios de las queserías, se encontró que 62 \% son familiares en línea directa, mientras que $38 \%$, aunque pueden ser menonitas, no tienen parentesco directo. Además, $67 \%$ de los empleados de las queserías son hijos del responsable o propietario de la fábrica de queso, mientras que el resto de los trabajadores no pertenecen a la familia.

Otro indicador importante es que, al momento del matrimonio, en la dote, a las mujeres les obsequian vacas. De tal forma que ellas son las propietarias de las mismas, así como de la leche; sin embargo, en el caso de las queserías, no tienen alguna participación ni colaboran de manera directa o indirecta en el proceso de producción del queso. Todo el esquema de negocio del queso, a pesar de ser cooperativa, se asienta en las bases de padres-hijos.

A continuación, se mencionarán algunos otros indicadores que muestran las características de las queserías en el noroeste de Chihuahua:

- Empleo: esta variable indica el tamaño de la agroindustria. En este estudio, se encontró que las queserías tienen una planta laboral de cinco empleados o menos, que representan $54.5 \%$ del total, lo que es un indicativo de que la mayoría son microempresas.

- Principal insumo: para la elaboración del queso, la leche proviene fundamentalmente de la producción de las colonias menonitas, ya que la ganadería de leche es parte de su cultura, 
aunque no es común observar grandes hatos, debido a que la producción proviene de pequeñas explotaciones. Parte de la producción la emplean para el autoconsumo y los excedentes, los comercializan a las queserías (un transporte pasa al pie de la finca para recoger los botes con el producto).

- Mercado: la comercialización del queso menonita en la región noroeste del estado de Chihuahua, se dirige a mercados mayoristas, principalmente en la Central de Abasto, restaurantes y autoservicios. En un poco más de $90 \%$, el producto se dirige a mercados de mayoreo, lo que indica con solidez la demanda del producto, ya sea porque los menonitas lo distribuyen en la ciudad en días específicos, o bien, lo entregan a intermediarios, quienes a su vez lo distribuyen en puntos de venta fuera de la región. Al momento de redactar este texto, se tuvo información de que buena parte de la producción de queso de la región es llevada a varias ciudades del estado de Sonora, como Agua Prieta, Hermosillo y Guaymas, además de los estados de Texas, Arizona y California, en el sur de Estados Unidos, aunque no se ha podido corroborar tal información.

\subsection{Perfil del consumidor}

De las personas encuestadas, más de $70 \%$ tenían nivel de escolaridad preparatoria o secundaria, de las cuales $56 \%$ eran de género femenino y $44 \%$, masculino. Se trató de hacer lo más equilibrada posible, sin embargo, la tendencia de mujeres en los puntos seleccionados fue más alta, debido a que son quienes, en su mayoría, acuden a efectuar las compras para la despensa. La actividad de los participantes en la encuesta fue: $38 \%$ eran empleados, $23 \%$ se dedicaban a las labores del hogar y $17 \%$ eran estudiantes, mientras que el resto abarca otras actividades, como comerciante, profesionista, profesor, actividades del campo, etcétera. Del total de encuestados, 57 \% correspondieron a Nuevo Casas Grandes, mientras que 43 \%, a Ciudad Juárez.

El $77 \%$ de los consumidores prefieren el producto por su sabor, pero llama la atención que 17 \% lo compran por costumbre, 
es decir, las otras marcas no tienen la penetración en el gusto de los consumidores como el queso menonita.

Aunque $70 \%$ de los consumidores del queso menonita afirmaron conocer la diferencia del queso menonita con otros, la mayoría (73 \%) mencionaron que es diferente por el sabor y casi $27 \%$, por la textura.

A pregunta expresa de si conocían el lugar de origen del queso menonita, las respuestas fueron variadas: desde que su origen era de los campos menonitas considerando toda una generalidad, pues cuando se les preguntaba si podían mencionar alguna en particular, la respuesta era negativa. Algunos sólo mencionaron Cuauhtémoc como el único asentamiento menonita. Esto implica que la mayoría de los consumidores desconocen el lugar de origen y/o producción del queso que se adquiere en mercados de Ciudad Juárez y que es prácticamente seguro que provienen de algún municipio de la región de Casas Grandes. Ello puede observarse mejor en la gráfica 1.

Gráfica 1. Lugar de origen del queso, según la opinión de los consumidores.

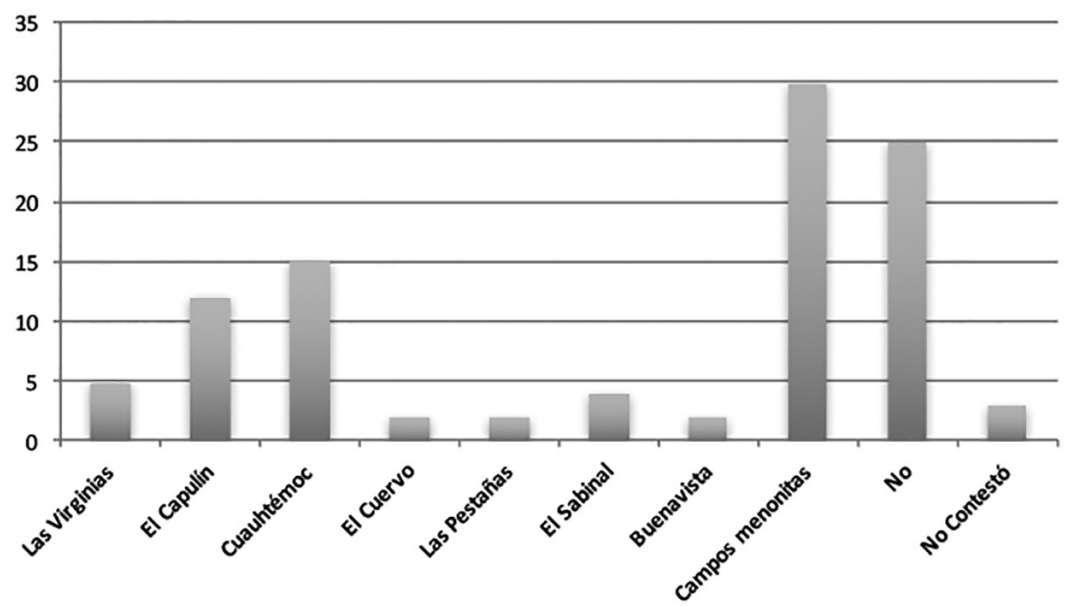

Fuente: elaboración propia (2016).

Los consumidores del queso menonita lo prefieren por su sabor y calidad, principalmente, aunque algunos lo distinguen por 
su textura, color y olor, tal como se muestra en la gráfica 2. Es relevante mencionar que $47 \%$ de los entrevistados mencionaron el sabor como la característica principal del queso menonita, mientras que 20 \% indicaron que, para ellos, la diferencia radica en el proceso, pues consideran que es más casero o artesanal, aunque para algunos fue interesante el comentario de que se corre un riesgo si no está bien elaborado o carece de normas sanitarias.

A pesar de que los consumidores pueden elegir entre diferentes presentaciones, no les incomoda que sea en rueda e, incluso, consideran que cualquiera de las dos presentaciones es buena. Es decir, $40 \%$ de los consumidores mencionan la compra de ambas presentaciones, en tanto que $35 \%$ optan más por la barra y el resto (25\%), se quedan con la de rueda. Cabe mencionar que las barras tienen diferentes presentaciones, las cuales van desde los 250 gramos hasta las de un kilo y medio, mientras que las ruedas son de tres y cinco kilos.

Gráfica 2. Características principales del queso menonita (sin degustación).

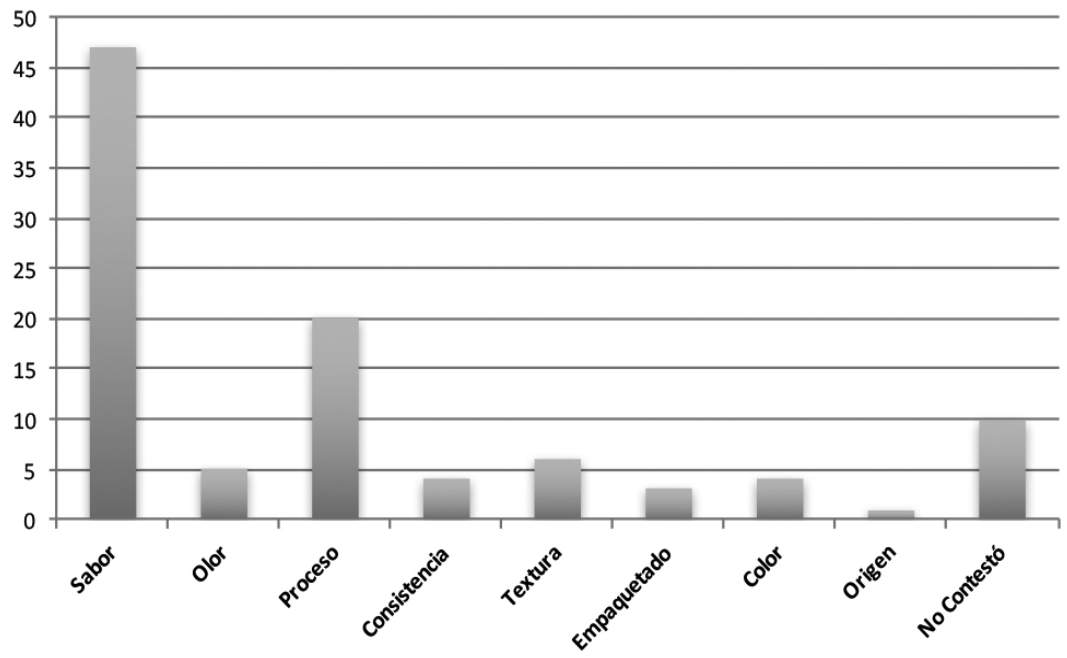

Fuente: elaboración propia (2016). 
La conducta de la compra del producto en las tiendas está directamente relacionada con la cantidad y frecuencia de la compra. Como ya se mencionó, la compra es, en promedio, semanal y se adquiere alrededor de medio kilo, pues $36 \%$ indicaron que compran el producto, mientras que $34 \%$ adquieren en el mismo periodo menos de un kilo y, aún más interesante, 28 \% de las personas encuestadas compran de uno a dos kilos por semana. Esto implica que, al menos, 97 \% de la población compra queso menonita.

En cuanto al lugar donde los consumidores prefieren comprar el queso, principalmente es en la tienda departamental, y posteriormente en el mercado y las queserías. Aunque es de mencionar que en Nuevo Casas Grandes existe una gran variedad de tiendas especializadas en la venta directa de los productores, quienes ofrecen los quesos y otras opciones lácteas como la mantequilla y la crema (18 \%). En Ciudad Juárez es más común la compra del queso en tiendas departamentales $(30 \%)$ o de autoservicio (40\%), pero ese tipo de queso es frecuentemente de las grandes empresas, que en los últimos meses comenzaron a introducirlo al mercado como marca. El resto prefieren comprarlo en el primer lugar donde lo encuentren y solo 2 \% tratan de comprarlo directamente con el productor, cuando visitan alguna colonia o en un local, que por lo regular se encuentra a la orilla de la carretera.

Esto, sin duda, es una oportunidad para los productores de queso, pues representa un mercado amplio con una demanda constante, porque no solo es identificado por el consumidor como un buen producto alimenticio, sino que está incorporado a la dieta cotidiana de los chihuahuenses, lo cual se muestra en la cantidad de platillos que incluyen este ingrediente. En el siguiente apartado, se mostrará con mayor detalle.

\subsection{Queso menonita, gastronomía y consumidores}

Respecto a la gastronomía, los encuestados mencionaron que su consumo es diario, el cual es utilizado en una amplia variedad de pla- 
tillos, ya sea más elaborados o en aquellos que se preparan de manera rápida o se sirven para calmar antojos.

Los principales platillos preparados que requieren queso, de acuerdo a la encuesta, son enchiladas, quesadillas, chiles rellenos, etcétera, además de los conocidos "burritos", para los cuales también puede usarse el queso asadero, que es circular y delgado como una tortilla, aunque más grasoso, pero que sí hace un poco de hebra, aunque no tan extensa ni firme como el queso Oaxaca, el cual es más cremoso y de color blanquizco. Curiosamente, el asadero es un queso típico regional y su producción se localiza, en mayor parte, en el municipio de Ahumada, población ubicada a $120 \mathrm{~km}$ al sur de Ciudad Juárez.

De ahí que al cuestionar sobre las segundas opciones cuando no se encuentra el queso menonita, se obtuvo que los consumidores mencionan al asadero como otro tipo de queso que compran (cerca de $27 \%$ ).

En relación con el nivel de consumo, se obtuvo que 29 \% de las personas comen queso en alguno de sus platillos, ya sea en tiempos de fuerte alimentación, en entremeses durante el día o en algún bocadillo. A ello se suma que $39 \%$ lo hacen, al menos, dos veces por semana, mientras que $19 \%$ incluyen el queso una vez a la semana. Es decir, si se suma cada uno de estos elementos, se obtiene que 87 \% de la población encuestada tiene una ingesta regular de queso en su dieta diaria. 
Gráfica 3. Preferencia de quesos por parte de los consumidores (con degustación).

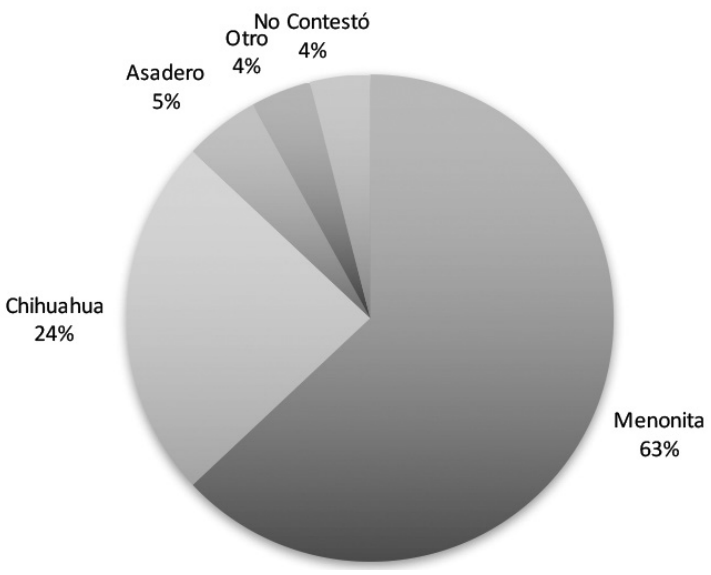

Fuente: elaboración propia (2017).

A la interrogante de cuál es, en su opinión, el queso más representativo del estado de Chihuahua, $63 \%$ mencionaron el queso menonita, $24 \%$ indicaron el queso chihuahua y $5 \%$ refirieron el asadero, mientras que $4 \%$ señalaron otros quesos no de la región y $4 \%$ prefirieron no contestar.

Para aclarar la discusión sobre las distinciones entre ambos quesos, los encuestados indicaron que el sabor es la principal diferencia (56\%), mientras que $17 \%$ mencionaron la textura y $7 \%$, el color. Los demás se distribuyeron entre olor, frescura y presentación. 
Gráfica 4. Características diferenciales del queso chihuahua vs. el queso menonita (con degustación).

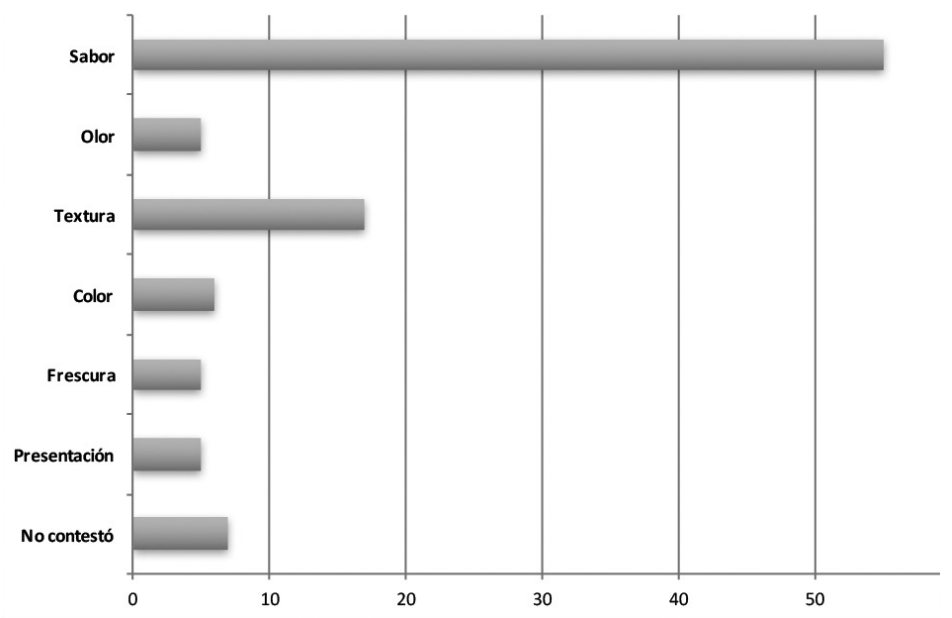

Fuente: elaboración propia (2017).

Luego de aplicar el cuestionario con base en la degustación de quesos, se obtuvo que sí existió una correcta identificación de los mismos, pues el resultado mostró que los encuestados sí identificaron correctamente el queso que se les dio a probar. Con ello puede comprenderse que si bien a ambos quesos se les asignan cualidades de buen sabor, para $68 \%$ el menonita fue considerado más sabroso, mientras que el resto señalaron al queso chihuahua. En cuanto al color, al queso chihuahua se le identificó por tener un color más amarillo, mientras que al menonita se le relacionó más con el color blanco. Respecto a la textura, el queso chihuahua se identificó con dureza, mientras que el menonita con suavidad.

Incluso, los encuestados respondieron que el mejor regalo para ofrecer a un extranjero como representativo de la región, es el queso menonita, pues $69 \%$ lo eligieron como su preferido, seguido de las tortillas de harina (16\%), el chile rojo (6\%) y la carne seca (6 \%). En relación con el motivo de su elección, que fue una respuesta abierta, respondieron que el sabor (33 \%), por tradición 
o muestra de su cultura (25\%), por ser su preferido (21\%) y por ser el más representativo (14\%).

Resulta interesante que si se considera como obsequio para algún amigo, igualmente el queso menonita vuelve a ser el favorito (59\%), mientras que ahora sí aparecen el queso chihuahua (26 $\%)$ y el asadero (8 \%).

Por tanto, ello indica que no sólo se puede identificar el queso menonita por sus características físicas, sino también por degustación, y principalmente se reafirma, de manera subjetiva, el aprecio que se tiene por el producto.

\section{CONCLUSIONES}

Con base en las preguntas que al comienzo del documento se plantearon, las conclusiones se ordenan de la siguiente manera:

El queso menonita definitivamente contribuye a la construcción de una subjetividad en el norte de México, pues el producto se toma como parte de la identidad local y del propio territorio. Aun cuando no se conozcan las diferencias entre el queso chihuahua y el queso menonita, los consumidores sí son capaces de identificarlos, por lo que se deben reforzar las diferencias para que no se confundan ambos productos. Además, en el sentido estricto de la subjetividad, al queso se le asignan elementos simbólicos de identidad con el territorio, pues al considerar más de la mitad de la población encuestada que el queso es un alimento de buen sabor y que tiene elementos culturales y tradicionales, como el trabajo, sin duda explica mucho el que sea uno de los productos gastronómicos que más se consumen en la región. Es decir, hay un apego y reconocimiento, así como, de manera sutil, un vínculo gastronómico con los miembros de las colonias menonitas, a quienes se les reconoce su aportación, y, por ello, se mantiene la denominación como el elemento diferenciador pero a la vez de identidad, debido a que también conlleva la significación de las dos principales migraciones que aún se mantienen evidentes en el estado de Chihuahua: la presencia de grupos religiosos como los mormones y los menonitas. Al combinarse ambos conocimien- 
tos y tradiciones, se pudo lograr un buen producto que, como se mencionó, es de origen definitivamente europeo y que ha sido adaptado a las condiciones locales, territoriales y ambientales de los menonitas.

En ese sentido, es necesario repensar de manera más seria la posibilidad de recuperar este proceso, analizar sus potencialidades y considerar las bondades, tanto para productores como para consumidores, pues en otras entidades de México y en Europa no necesariamente la pasteurización de la leche refleja una buena calidad en el proceso de elaboración de los quesos. En todo caso es necesario pensar en, al menos, tres niveles: en primer lugar, los procesos, las buenas prácticas del manejo de ganado y sus productos; un segundo nivel será diseñar una estrategia de comunicación donde pueda incluirse la importancia de los productos locales que brindan esos elementos de orgullo y satisfacción. Incluso, hasta como elemento de turismo gastronómico, donde se puedan identificar las zonas o regiones de producción. Por el momento, en ciudad Cuauhtémoc las iniciativas, a través del museo Menonita, están comenzando a tener éxito, pero también podrían establecerse elementos más específicos para las colonias de esa región. El tercer nivel tiene que ver con los consumidores, ya que, como fue observado con los encuestados, existe una clara identificación entre las diferencias de ambos quesos, por lo que podría ser conveniente proporcionar mayor información para que exista una mejora en los productos.

Con ello, las autoridades sanitarias y de desarrollo social podrán considerar esta posibilidad, para que se pueda revisar el valor de este tipo de productos tradicionales y propios de una región.

Por otra parte, respecto a las características agroindustriales que se muestran en el noroeste del estado de Chihuahua, a partir de la producción del queso menonita, puede decirse que este es un producto que es canalizado principalmente al mercado de Ciudad Juárez. La mano de obra es relativamente barata, debido a que, principalmente, son familiares de los dueños, y cuando requieren personal contratan empleados de la misma colonia y en casos excepcionales a empleados no menonitas. 
De las tendencias de consumo existentes en la región, pudo observarse que:

Derivado de las encuestas aplicadas, se concluye que en las poblaciones de Ciudad Juárez y Nuevo Casas Grandes, el principal queso consumido es el menonita, seguido por el asadero; ambos quesos son identificados por la población como regionales y típicos del estado de Chihuahua.

Los consumidores prefieren el queso menonita principalmente por su sabor y calidad y el principal platillo que preparan con este producto son las enchiladas.

En lo referente a las oportunidades del mercado, con base en el modelo FODA (Fortalezas-Oportunidades-Debilidades-Amenazas), que ayuda a sintetizar de manera muy precisa los elementos y categorías que se deben considerar, se obtiene que, al menos, dentro de las lecherías y queserías de la región noroeste del estado de Chihuahua, se pueden identificar las siguientes fortalezas:

- Unidad de los productores de leche en las colonias menonitas

- Apoyo entre queseros para resolver problemas técnicos o de materias primas

- Accesos a financiamientos por parte de órganos financieros

- Procesos de producción estandarizados

- Entrega del producto terminado en los puntos de venta

Las oportunidades para estas empresas, productores y comercializadores pueden considerarse amplias, principalmente porque hay un buen producto, no existen problemas para colocarlo y, además, se cuenta con la mejor publicidad: la recomendación de boca en boca entre los consumidores, aunado a que muchas personas que viven en Ciudad Juárez tienen familiares o conocidos de la región; a lo largo de esta investigación, se pudo observar lo siguiente:

- Producto incorporado en la dieta cotidiana de la región

- Creciente demanda del producto en el mercado regional 
- Producto relacionado con la cultura y la tradición de Chihuahua

- Contexto legal de apoyo con base en las denominaciones de origen

- Identificación del producto por medio de un plan mercadológico

- Nostalgia del campo y sus productos

Respecto a las amenazas, estas existen para todos los productores, en especial para aquellos que aún no tienen el registro de marca del producto y lo venden a intermediarios, quienes a su vez se adjudican el nombre y el registro, por lo que el comprador no se preocupa por conocer una marca ni quiénes son los productores. De tal forma que las amenazas serán:

- Otros productos de baja calidad usando análogos y competencia desleal

- Comercialización de quesos elaborados con grasa vegetal y caseinato, por parte de productores no menonitas, quienes les dan la denominación de menonita, lo cual será una mala referencia para los consumidores

La Secretaría de Salud, a través de la Comisión Estatal para la Protección contra Riesgos Sanitarios (Coespris), ejerce presión para la pasteurización de la leche y los amenaza con el cierre de las queserías.

En cuanto a las debilidades, es necesario atender todos estos elementos, ya que a lo largo del proceso de producción se tienen identificadas una serie de problemas que, para algunos, no necesariamente representan una dificultad, pues existen situaciones que se resuelven sin atender la comercialización o áreas específicas de la mercadotecnia. Esto principalmente se detectó con los productores y algunos queseros, quienes prefieren apoyarse en los intermediarios en vez de asumir los costos y riesgos de desplazar el producto a los puntos de venta o buscar la forma de su registro: 
Por lo que las debilidades serán:

- Falta de registro de marca ante el Instituto Mexicano de la Propiedad Industrial (IMPI)

- Incremento de intermediarios para el desplazamiento del producto de las centrales de abasto a las tiendas al menudeo

- Procesos de producción artesanales

- Falta de estrategias de comunicación y/o difusión sobre el producto

- Estrategias para la introducción y posicionamiento de la marca

- Desarrollo de emblema o sello de producto tradicional

- Desarrollo de una narrativa aspiracional (branding), que refuerce la identidad local, regional y estatal.

\section{BIBLIOGRAFÍA}

Belletti, G. (2003): Le denominazioni geografiche nel supporto all'agricoltura multifunzionale. In Politica Agricola Internazionale, 4, 81-102.

Belletti, G., Brunori, G., Marescotti, A., Pacciani, A., \& Rossi, A. (2006).

Il processo di valorizzazione delle produzioni agroalimentari tipiche. In B. Rocchi, \& D. Romano (a cura di), Tipicamente buono. Concezioni di qualità lungo la filiera dei prodotti agro-alimentari in Toscana. Milano: Franco Angeli, 175-198.

Benavente Chorres, H. (2008). El contrato de know how o de provisión de conocimientos técnicos: aspectos a ser considerados para su regulación normativa. Revista Ius et Praxis, 2, 407-457. Recuperado de http://www.scielo.cl/pdf/iusetp/v14n2/art12.pdf

Drake, M. A. (2004). Defining Dairy Flavor. Journal of Dairy Science, 87(4), 777-784.

Instituto Nacional de Estadística y Geografía (Inegi) (2015). Encuesta Mensual de la Industria Manufacturera (EMIM). Sistema de Clasificación Industrial de América del Norte (SCIAN) 2007, p. 426. Recuperado el 28 de abril de 2016.

Llera Pacheco, F. J., \& Bautista Flores, E. (2013). Comunidades menonitas de México y Brasil: influencia y aportaciones. México: 
Universidad Autónoma de Ciudad Juárez/Universidade Estadual de Ponta Grossa/Programa de Mejoramiento del Profesorado.

López, J. A., \& Vargas, C. (2011). El queso de Chihuahua: queso chihuahua. Chihuahua Hoy. México: Universidad Autónoma de Ciudad Juárez. Recuperado de http://webcache.googleusercontent. $\mathrm{com} /$ search?q=cache:rQsnu4Jt9F4J:elibros.uacj.mx/omp/index. php/publicaciones/catalog/download/63/58/347-1+\&cd=5\&hl=es\& $\mathrm{ct}=\mathrm{clnk} \& \mathrm{gl}=\mathrm{mx} \& \mathrm{client}=$ safari

Programa 25. Queso menonita. Recuperado el 18 de abril de 2016 de https://www.youtube.com/watch?v=ohS0E5Lzs5w.

Ranaboldo, C., Venegas, C., Quirós, R., Báez Lacayo, L., Rivas Villamizar, N., Palma, V., \& Yaksic Soulé, A. (2010). Desarrollo de los agronegocios y la agroindustria rural en América Latina y el Caribe: conceptos, instrumentos y casos de cooperación técnica (ICA E20-58). Instituto Interamericano de Cooperación para la Agricultura.

[s/a]. Logra queso chihuahua NOM para fabricación de sus productos. XHEPL 91.3 FM • 550 AM. Recuperado el 26 de abril de 2016 de http://www.xepl.com.mx/completa1.php?i=59809

Sánchez Carlos, O., \& Bautista Flores, E. (2016). Entrevista a Abraham Petter. Ciudad Cuauhtémoc.

Sánchez Carlos, O., Bautista Flores, E, \& Cervantes Escoto, F. (2016). Queso menonita. Producto ícono de los menonitas de Chihuahua. Primer Simposio Nacional de los Quesos Tradicionales (pp. 78-87). Chapingo, México.

Secretaría de Agricultura, Ganadería, Desarrollo Rural, Pesca y Alimentación- Servicio de Información Agroalimentaria y Pesquera (Sagarpa-SIAP) (2016). Panorama de la leche en México. Recuperado de http://infosiap.siap.gob.mx/opt/boletlech/Brochure\%20 leche_Diciembre2016.pdf

Van Hekken, D. L., Drake, M. A., Molina Corral, F. J., Guerrero Prieto, V. M., \& Gardea, A. A. (2006). Mexican Chihuahua Cheese: Sensory Profiles of Young Cheese. Journal of Dairy Science, 89(10), 3729-3738. 
Velarde, I. (2012). La construcción social de productos agroalimentarios típicos en procesos de desarrollo territorial local: disputas entre saberes teóricos y saberes prácticos. Mundo Agrario: Revista de Estudios Rurales, 12(24). Recuperado de http://www.redalyc. org/articulo.oa?id=84525452014

Villegas de Gante, A., \& Cervantes Escoto, F. (2011). La genuinidad y tipicidad en la revalorización de los quesos artesanales mexicanos. Estudios Sociales, 19(38), 146-164. 